\title{
Autoimmune hearing loss and ulcerative colitis
}

\author{
Renato Prescinotto ${ }^{1}$, Raul Vitor Rossi Zanini, Priscila \\ Bogar Rapoport ${ }^{3}$, Carlos Augusto Anadão, Renata \\ Ribeiro de Mendonça ${ }^{5}$, Roberta Borges Novais Petrilli ${ }^{6}$
}

Keywords: hearing loss, autoimmune diseases, colitis, ulcerative.

\section{INTRODUCTION}

McCabe first described autoimmune hearing loss in $1979^{1}$.

Summers and Harker described the first case of dysacusis associated with ulcerative colitis in 1982. Since then other cases have been reported ${ }^{2,3}$.

\section{CASE PRESENTATION}

This is a male 23-year-old patient presenting with right tinnitus, ipsilateral hypoacusis and dizziness following three months of diarrhea and weight loss. The patient was taking deflazacort and budesonide, which improved his intestinal, hearing and postural symptoms, but not the tinnitus.

Initial audiometry revealed mild right sensorineural dysacusis.

Laboratory exams included a positive ANF, an increased C-reactive protein, mucoproteins and HSV. Otoneurology suggested a right peripheral vestibular deficit syndrome. Magnetic resonance imaging was normal.

Colonoscopy showed colon and rectal hyperemia with erosions and ulcers, distal transverse colon and distal rectal stenosis; pathology disclosed non-specific
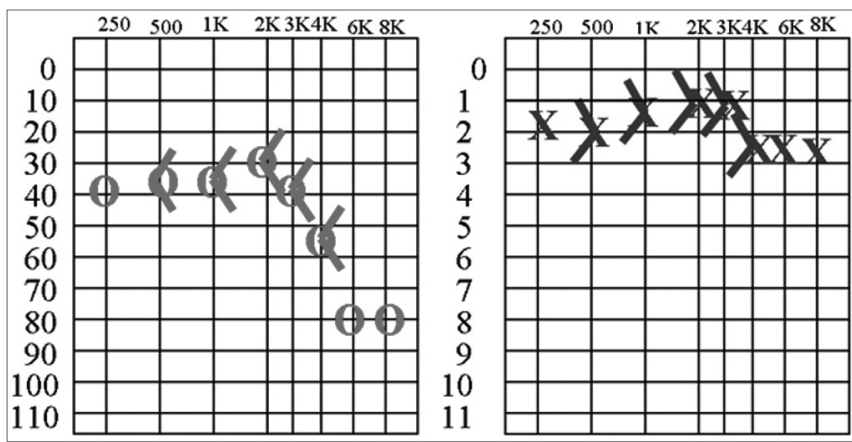

chronic rectocolitis.

The patient used medication during one month and remained clinically stable for a further three months before recurring. Audiometry revealed a right auditory decline in all frequencies, mostly for higher frequency sounds. (Figure 1)

Prednisone (60 mg per day) was introduced, with clinical improvement, after which the dose was reduced to $20 \mathrm{mg}$ per day. Follow-up audiometry revealed audiometric improvement, especially at 1 and $2 \mathrm{KHz}$. Mild asymptomatic left dysacusis for lower frequency sounds was also observed.

The patient remained with occasional right hypoacusis and constant tinnitus, although this did not interfere in his daily activities; intestinal symptoms also regressed. At present the patient is taking mesalazine and prednisone.

\section{DISCUSSION}

Sensorineural hearing loss has frequently been described in association with a variety of autoimmune diseases ${ }^{3-6}$.

Although the pathophysiology of sensorineural deafness remains obscure, the autoimmune nature of the disease strengthens some proposed theories that and progression $^{1-6}$.

These forms of dysacusis usually present as a subclinical condition or as severe bilateral hearing loss, such as sudden deafness 5 .

There are no specific tests to establish the diagnosis of autoimmune dysacusis. A few proteins are being isolated and may have a strong connection with the autoimmune condition, such as the HSP 70 protein, although these findings are of little practical value at present ${ }^{5,6}$.

Treatment includes aggressive use of immunosuppressants, usually corticosteroids. Other immunosuppressants have also been suggested by some authors ${ }^{3-5}$.

The differential diagnosis is required for cases of sensorineural dysacusis. However, exams will not necessarily confirm a specific etiology. In such cases a treatment may be given as a test to confirm the diagnosis.

\section{FINAL COMMENTS}

Autoimmune dysacusis is one of the few sensorineural conditions in which clinical improvement may be obtained, as long as the diagnosis is promptly made and treatment is adequately employed.

\section{REFERENCES}

1. McCabe B. Autoimmune hearing loss. Ann Otol Rhinol Laryngol 1979;88:585.

2. Summers RW, Harker L. Ulcerative colitis and sensorineural hearing loss: is there a relationship? J Clin Gastroenterol 1982;4:251.

3 Weber RS, Jenkins HÁ, Coker NJ. Sensorineural hearing loss associated with ulcerative colitis. Arch Otolaryngol 1984;110:810-2.

4 Hollanders D. Sensorineural deafness - a new complication of ulcerative colitis? Postgrad Med J 1986;62:753-5

5 Herdman RC, Hirari M, Ramsden RT. Autoimmune inner ear disease and ulcerative colitis J Laryngol Otol 1991;105(4):330-1.

6 Kumar BN, Walsh RM, Wilson OS, Carlin WV. Sensorineural hearing loss and ulcerative colitis. J Laryngol Otol 1997;111(3):277-8.

Figure 1. Pure tone and vocal audiometry during recurrence of the condition.

$1 \mathrm{MD}$, Otorhinolaryngology Resident - Medical School of the ABC

$2 \mathrm{MD}$, Otorhinolaryngology Resident - Medical School of the ABC

3 MD, Otorhinolaryngologist, PhD in Otorhinolaryngology - FMUSP. Full Professor of Otorhinolaryngology - Medical School of the ABC.

4 MD, Otorhinolaryngologist, Assistant Professor of Otorhinolaryngology - Medical School of the ABC

5 MD, Otorhinolaryngology Resident - Medical School of the ABC

${ }^{6} \mathrm{MD}$, Otorhinolaryngology Resident - Medical School of the ABC..

Paper submitted to the RBORL - SGP (Publication Management System of the Brazilian Journal of Otorhinolaryngology) on March 11th, 2005 and approved on May 31 st, 2006. Paper submitted to the ABORL-CCF SGP (Management Publications System) on March 11th, 2005 and accepted for publication on May 31 st, 2006. 